\title{
Bladder-conserving Approach in Radical Treatment of Patients With Bladder Cancer - A Single-institution Experience
}

\author{
WOJCIECH MAJEWSKI ${ }^{1}$, JAROSLAW NIECKULA ${ }^{2}$, TOMASZ DWORZECKI ${ }^{1}$ and LESZEK MISZCZYK ${ }^{1}$ \\ ${ }^{1}$ Radiotherapy Department, Maria Sklodowska-Curie National \\ Research Institute of Oncology, Gliwice Branch, Gliwice, Poland; \\ ${ }^{2}$ Single-Day Chemotherapy Ward, Maria Sklodowska-Curie National \\ Research Institute of Oncology, Gliwice Branch, Gliwice, Poland
}

\begin{abstract}
Aim: To evaluate our experience with radical radiotherapy and chemotherapy in patients with muscleinvasive bladder cancer. Patients and Methods: The study consisted of 27 patients treated with cisplatin-based chemoradiation (CCRT), 48 treated with radiation alone $(R T)$, and 42 with locally advanced disease treated with neoadjuvant chemotherapy and radiation (neoCRT). Results: The incidence of acute grade 3 or more genitourinary $(G U)$ toxicity in the RT, CCRT and neoCRT groups was: $25 \%, 11 \%$ and $19 \%$, respectively $(p=0.029)$. The 3-year freedom from grade 2 or more GU toxicity was: $81 \%, 89 \%, 54 \%$, respectively $(p=0.36)$. The long-term outcomes of 3-year local control, overall survival, and disease-free survival were as follows: RT group: $74 \%, 61 \%$ and 55\%; CCRT group: $76 \%, 76 \%$ and $56 \%$; neoCRT group: $31 \%, 43 \%$ and $18 \%$, respectively. Conclusion: The preferable bladder-conserving approach is CRT, however RT alone might also be an option for appropriately selected patients. NeoCRT for those with locally advanced tumors remain unsatisfactory; adequate selection of patients for radical treatment is of importance.
\end{abstract}

Muscle-invasive bladder cancer is still a great challenge for radical treatment. The gold-standard approach of 'radical cystectomy' is now being questioned as the best solution, and bladder-conserving tri-modality therapy is currently considered a comparable alternative (1). Such treatment comprises

This article is freely accessible online.

Correspondence to: Wojciech Majewski, MD, Ph.D., Radiotherapy Department, Maria Sklodowska-Curie National Research Institute of Oncology, Gliwice Branch, Wybrzeze Armii Krajowej 15, 44100 Gliwice, Poland. Tel: +48 322788001, Fax: +48 322788001, e-mail:wmajewski1@poczta.onet.pl

Key Words: Bladder cancer, radiotherapy, chemoradiotherapy, neoadjuvant chemotherapy, bladder sparing. transurethral resection (TURBT) and concurrent chemoradiation (CCRT), mainly based on cisplatin, however, other options using mitomycin and 5-fluorouracil or gemcitabine can also be utilized $(2,3)$. Treatment and fractionation schedules differ in the literature, however, once-daily irradiation is commonly adopted and was shown not to be inferior to twice-a-day irradiation $(2,4)$. Although chemoradiation seems to be more efficient than radiation alone (RT), revealing some improvement in local or regional control but without significant impact on overall survival, direct comparisons in randomized trials are scarce $(3,5)$. Large retrospective studies, however, show the benefits of chemoradiation over RT alone in terms of overall survival (OS) (6).

Patients with more advanced bladder tumors are a real challenge. It is uncertain if they can be treated radically and a large proportion of them should perhaps undergo palliative treatment. RT alone probably does not suffice and it is an open question if a CCRT with low-doses of radio-enhancing cytostatics, often administered in monotherapy, is enough to kill the micrometastatic spread of tumor cells. Even in the case of a good local response, the chance of a long-lasting cure may be questionable. In such patients, neoadjuvant chemotherapy may potentially be a valuable option (7).

Hence, the aim of this study was to assess the outcome of patients with muscle-invasive bladder cancer treated with different bladder-conserving methods (TURB plus RT alone, or CRT) which changed over the study period. Additionally, a group with more advanced locally/regionally bladder cancer treated with neoadjuvant chemotherapy and RT (neoCRT) was evaluated.

\section{Patients and Methods}

Patients. The study group consisted of three separate subgroups of patients with muscle-invasive urothelial bladder cancer treated with bladder-conserving therapy: CCRT group: a prospective group treated between 2010 and 2017 with tri-modality treatment of TURBT plus CRT. RT group: a retrospective group treated in earlier years (between 2006 and 2010) when TURBT and RT alone was a 
standard treatment for such patients; neoCRT: a retrospective group of patients with more advanced tumors treated between 2005 and 2016 with neoadjuvant chemotherapy and subsequent RT.

The groups were somewhat different in the treatment intention and characteristics. The RT group consisted of patients referred for radiation because of patient preference, or contraindications to surgery. In those years, TURBT plus RT was the main method of treatment at our Institution.

The CCRT group consisted of patients treated in subsequent years when the protocol of tri-modality treatment had been implemented.

The last group consisted mostly of patients with advanced and larger-volume tumors. In order to exclude a possible bias related to the evaluation of only patients undergoing the whole treatment course, all patients who were eligible for neoadjuvant chemotherapy were included in the intent-to-treat basis irrespective of the response to chemotherapy and further treatment.

Although the groups were not prospectively selected, we wanted to compare the outcome between the RT group, which represented our previous standard, and the group treated with CCRT, which is currently used. Owing to the fact that the RT and CCRT groups were not well balanced with respect to T-stage, only patients with $\mathrm{T} 2$ and $\mathrm{T} 3$ stages were selected from the RT group for further analysis and comparison with CCRT group. A detailed statistical comparison with the neoCRT group was not performed as it was a different patient population (with more advanced disease).

The RT group consisted of 48 patients, the CCRT group of 27 patients and the neoCRT group of 42 patients. The clinical characteristics of the study groups are presented in Table I.

Although there was some imbalance with respect to the tumor grade, the differences between the RT group and the CCRT group with respect to those with high-grade (or grade 3) tumors were insignificant $(p=0.24)$.

However, due to the fact the patients in the CCRT group had to be fit in order to undergo CRT, that group might have had a better comorbidity profile.

Treatment. RT group: Patients were treated with 3D conformal intensity-modulated RT techniques using a linear accelerator with 6$20 \mathrm{MV}$ photons. Clinical target volume 1 (CTV1) was pelvic lymph nodes and planning target volume 1 (PTV1) was constructed by adding approximately a $0.5 \mathrm{~cm}$ margin around the CTV1. The CTV2 was the whole bladder, and PTV2 was created by adding a nonuniform margin of approximately $1.0-1.2 \mathrm{~cm}$ in all directions except for superior and anterior where it was about $1.8-2.0 \mathrm{~cm}$. In almost all cases, pelvic lymph nodes were irradiated to a total dose of $44 \mathrm{~Gy}$ with 2 Gy per fraction. The boost to the PTV2 was administered to a total dose of 66-70 Gy with a dose per fraction of 2 Gy.

CCRT group: The contouring and irradiation rules were similar to the RT group, but the final boost to the PTV2 was given to the total dose of 64-66 Gy. Concurrent chemotherapy was given every 3 weeks with a cisplatin dose of $80 \mathrm{mg} / \mathrm{m}^{2}$. In total, three cycles of chemotherapy were planned during RT. In $40 \%$ of patients, chemotherapy was planned in other schedules, usually with a weekly cisplatin schedule of $30 \mathrm{mg} / \mathrm{m}^{2}$ given six times during RT.

NeoCRT: This was mainly based on gemcitabine with platinum scheme or methotrexate, vinblastine, doxorubicin and cisplatin (MVAC). In three patients, one cycle of neoadjuvant chemotherapy was used $(7 \%)$, in 17 patients $(41 \%)$ two cycles, in 16 patients $(38 \%)$ three cycles, and in six patients (14\%) four cycles were applied. Disease in one patient progressed during neoadjuvant
Table I. The clinical characteristics of the study groups.

\begin{tabular}{lccc}
\hline Clinical factor & RT $(\mathrm{n}=48)$ & CCRT $(\mathrm{n}=27)$ & NeoCRT $(\mathrm{n}=42)$ \\
\hline Age, years & & & \\
Mean \pm SD (range) & $67 \pm 8.4(49-87)$ & $65 \pm 8.0(44-76)$ & $63 \pm 6.5(49-75)$ \\
Gender & & & \\
M & $39(81 \%)$ & $21(78 \%)$ & $35(83 \%)$ \\
F & $9(19 \%)$ & $6(22 \%)$ & $7(17 \%)$ \\
T-Stage, n (\%) & & & \\
T2 & $37(77 \%)$ & $20(74 \%)$ & $11(26 \%)$ \\
T3 & $11(23 \%)$ & $7(26 \%)$ & $15(36 \%)$ \\
T4 & $0 \%$ & $0 \%$ & $16(38 \%)$ \\
N-Stage, n $(\%)$ & & & \\
N0 & $48(100 \%)$ & $27(100 \%)$ & $33(79 \%)$ \\
N+ & 0 & 0 & $9(21 \%)$ \\
Grade, n (\%) & & & $4(10 \%)$ \\
G1 & $6(12.5 \%)$ & 0 & $9(21 \%)$ \\
G2 & $18(37.5 \%)$ & $7(26 \%)$ & $18(43 \%)$ \\
G3 & $12(25 \%)$ & $5(19 \%)$ & 0 \\
LG & $4(8 \%)$ & $6(22 \%)$ & 0 \\
HG & $5(10 \%)$ & $9(33)$ & $11(26 \%)$ \\
Missing data & $3(6 \%)$ & 0 & \\
\hline
\end{tabular}

RT: Radiotherapy alone; CCRT: cisplatin-based chemoradiation; NeoCRT: neoadjuvant chemotherapy and radiation. *The 3-step grading system of differentiation (grade 1-3) has been gradually replaced by the 2-step (LG: low grade, HG: high grade) system in later years of the study.

chemotherapy and was referred to symptomatic treatment. Among others, in five patients (12\%) progression was observed after neoadjuvant chemotherapy and those patients were referred to palliative RT. Radical RT was therefore administered to 36 patients (82\%). The parameters of RT are reported only for this subgroup (Table II) but the clinical characteristics are presented for the whole neoCRT group. RT was performed similarly to the rules for the RT group. Daily 2D-2D kV image guidance was performed with respect to the pelvic bones from 2008. In earlier years, portal imaging was performed before administration of the first treatment fraction.

The higher percentage of patients treated with dynamic techniques in the CCRT group is related to the more common utilization of such techniques in the later years of the study. The lower doses of RT given in the CCRT group than in the RT group are due to the rigid dose specification in the patient protocol for the former group.

Toxicity and adverse events. The tolerance of radiotherapy was assessed with the RTOG scale (8). Genitourinary (GU) and gastrointestinal (GI) toxicities were evaluated. Additionally, for patients treated with CCRT, hematological toxicity was evaluated during the treatment with the Common Terminology Criteria for Adverse Events (CTCAE) v3.0 (9). Acute toxicity was assessed as a crude rate of maximum toxicity during the radiation course. Late toxicity was assessed with a crude rate and with an actuarial method focusing on patients with grade 2 or more GU or GI toxicity. The time to the development of late toxicity was calculated from the beginning of RT.

Outcome endpoints. The long-term outcome was evaluated in terms of local control (LC), OS and disease-free survival (DFS), 
Table II. The parameters of radiotherapy in the studied groups.

\begin{tabular}{|c|c|c|c|}
\hline Parameter & $\begin{array}{c}\mathrm{RT} \\
(\mathrm{n}=48), \\
\mathrm{n}(\%)\end{array}$ & $\begin{array}{c}\text { CCRT } \\
(\mathrm{n}=27), \\
\mathrm{n}(\%)\end{array}$ & $\begin{array}{c}\text { NeoCRT } \\
(\mathrm{n}=36), \\
\mathrm{n}(\%)\end{array}$ \\
\hline \multicolumn{4}{|c|}{ Elective pelvic lymph nodes RT } \\
\hline Yes & $45(94 \%)$ & $27(100 \%)$ & $34(94 \%)$ \\
\hline No & $3(6 \%)$ & 0 & $2(6 \%)$ \\
\hline \multicolumn{4}{|l|}{ RT technique pelvis* } \\
\hline 3DCRT & $19(42 \%)$ & $3(11 \%)$ & $22(65 \%)$ \\
\hline Dynamic (IMRT, VMAT) & $26(58 \%)$ & $24(89 \%)$ & $12(35 \%)$ \\
\hline \multicolumn{4}{|l|}{ RT technique boost } \\
\hline 3DCRT & $37(77 \%)$ & $5(19 \%)$ & $23(64 \%)$ \\
\hline Dynamic (IMRT, VMAT) & $11(23 \%)$ & $22(81 \%)$ & $13(36 \%)$ \\
\hline \multicolumn{4}{|l|}{ Total radiation dose } \\
\hline 60-64 Gy & $1(2 \%)$ & $12(44 \%)$ & $4(11 \%)$ \\
\hline 66-68.4 Gy & $17(35 \%)$ & $15(56 \%)$ & $10(28 \%)$ \\
\hline 68.4-70.2 Gy & $28(58 \%)$ & 0 & $19(53 \%)$ \\
\hline Not completed $(<60 \mathrm{~Gy})$ & $2(4 \%)$ & 0 & $3(8 \%)$ \\
\hline
\end{tabular}

3DCRT: 3D Conformal radiotherapy; IMRT: intensity-modulated radiotherapy; RT: radiotherapy alone; CCRT: cisplatin-based chemoradiation; NeoCRT: neoadjuvant chemotherapy and radiation; VMAT: volumetric-modulated arc therapy. *Three patients in the RT-only group and two patients in the neoCRT group had irradiation to the bladder alone.

calculated with an actuarial method using Kaplan-Meier estimation. The time was calculated from the beginning of radiotherapy in the RT and CCRT groups, and from the beginning of neoadjuvant chemotherapy in the neoCRT group. Local failure was defined as pathologically confirmed persisting tumor after RT or local recurrence after tumor remission. In the neoCRT group, it was additionally loss to follow-up, shortly after RT before the possibility of an adequate response assessment, or non-completion of the whole planned treatment course (for instance, no radical RT or noncompletion of radical RT after neoadjuvant chemotherapy). The DFS was defined as no local, regional or distant progression or death irrespective of cause.

The incidence of acute toxicity was compared with the chisquared test, while late toxicity and long-term endpoints were evaluated with the Kaplan-Meier method and compared with the log-rank or Cox-Mantel test.

\section{Results}

The median follow-up was 6 years for the RT, 2 years for the CCRT and 2.5 years for the neoCRT groups.

Acute toxicity. The incidence and severity of acute GU and GI toxicity are presented in Table III.

Acute GU toxicity was similar for the RT and the neoCRT groups. However, only patients in the RT group developed serious grade 4 toxicity, in two cases; in one of them, RT was terminated at $60 \mathrm{~Gy}$ dose, the other patient had a 12-day gap and then treatment was continued. Patients in the CCRT group tolerated RT very well with significantly less acute
Table III. Acute radiotherapy-related toxicity among the treatment groups.

\begin{tabular}{lcccc}
\hline Acute toxicity & Grade & $\begin{array}{c}\text { RT group, } \\
\text { n (\%) }\end{array}$ & $\begin{array}{c}\text { CCRT group, } \\
\text { n (\%) }\end{array}$ & $\begin{array}{c}\text { NeoCRT } \\
\text { group, n (\%) }\end{array}$ \\
\hline Genitourinary & 0 & $7(15 \%)$ & $5(19 \%)$ & $5(14 \%)$ \\
& 1 & $7(15 \%)$ & $10(37 \%)$ & $8(22 \%)$ \\
& 2 & $22(46 \%)$ & $9(33 \%)$ & $16(44 \%)$ \\
Gastrointestinal & 3 & $10(21 \%)$ & $3(11 \%)$ & $7(19 \%)$ \\
& 4 & $2(4 \%)$ & 0 & 0 \\
& 0 & $24(50 \%)$ & $18(67 \%)$ & $24(67 \%)$ \\
& 1 & $11(23 \%)$ & $7(26 \%)$ & $9(25 \%)$ \\
& 2 & $13(27 \%)$ & $2(7 \%)$ & $2(6 \%)$ \\
& 3 & 0 & 0 & 0 \\
& $4 *$ & 0 & 0 & $1(3 \%)$ \\
\hline
\end{tabular}

RT: Radiotherapy alone; CCRT: cisplatin-based chemoradiation; NeoCRT: neoadjuvant chemotherapy and radiation. *One patient developed ileus during radiotherapy; pharmacological treatment was introduced and radiotherapy was finished on a 30 Gy dose.

Table IV. Late radiation-related toxicity among the treatment groups*.

\begin{tabular}{lcccc}
\hline Acute toxicity & Grade & $\begin{array}{c}\text { RT group, } \\
\text { n (\%) }\end{array}$ & $\begin{array}{c}\text { CCRT group, } \\
\text { n (\%) }\end{array}$ & $\begin{array}{c}\text { NeoCRT } \\
\text { group, n (\%) }\end{array}$ \\
\hline Genitourinary & 0 & $29(60 \%)$ & $19(70 \%)$ & $13(36 \%)$ \\
& 1 & $6(12 \%)$ & $4(15 \%)$ & $11(30 \%)$ \\
& 2 & $4(8 \%)$ & $2(7 \%)$ & $4(11 \%)$ \\
Gastrointestinal & 3 & $4(8 \%)$ & 0 & $3(8 \%)$ \\
& 4 & $1(2 \%)$ & 0 & 0 \\
& 0 & $39(81 \%)$ & $23(85 \%)$ & $31(86 \%)$ \\
& 1 & $3(6 \%)$ & $1(4 \%)$ & 0 \\
& 2 & $1(2 \%)$ & 0 & 0 \\
\hline
\end{tabular}

RT: Radiotherapy alone; CCRT: cisplatin-based chemoradiation; NeoCRT: neoadjuvant chemotherapy and radiation. *For four patients from the RT group, two patients from the CCRT group and five patients from the neoCRT group, follow-up was too short to reliably assess late toxicity.

grade 2 or more GU toxicity as compared to the RT group $(p=0.029)$. The differences between other groups were statistically insignificant.

Acute GI toxicity was similar for the CCRT and neoCRT groups. On the other hand, patients in the RT group experienced more pronounced acute GI toxicity. The rate of grade 2 or more acute GI toxicity was $27 \%$ as compared to $7 \%$ in the CCRT group $(p=0.014)$ and $8 \%$ in the neoCRT group $(p=0.047)$. The differences between the latter two groups were statistically insignificant.

In the CCRT group, hematological tolerance during treatment was very good. Only one case of grade 3 toxicity was reported. The higher rate of grade 2 toxicity for leucocyte count 


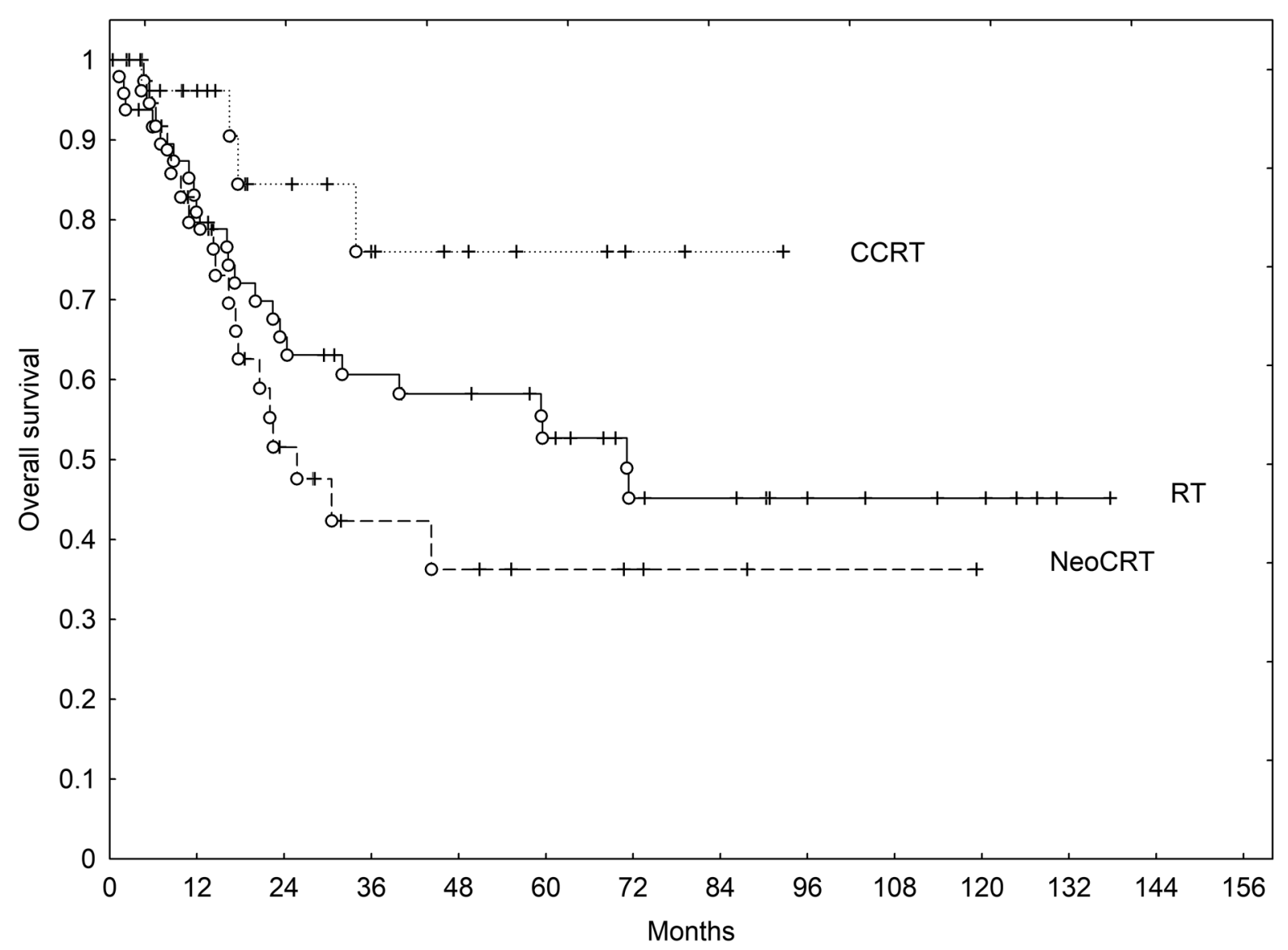

Figure 1. Overall survival of 27 patients treated with cisplatin-based chemoradiation (CCRT), 48 treated with radiation alone (RT), and 42 with locally advanced disease treated with neoadjuvant chemotherapy and radiation (neoCRT).

(44\%) than for neutrophil count (30\%) was probably due to an additional decrease in the lymphocyte count, which is a typical observation for patients given pelvic irradiation (10).

Late toxicity. The crude incidence of late toxicity by treatment group is presented in Table IV.

The 3-year freedom from grade 2 or more GU toxicity was: $81 \%, 89 \%, 54 \%$ in the RT, CCRT and neoCRT groups, respectively $(p=0.36)$. At 3 years, freedom from late grade 2 or more GI toxicity was $91 \%, 100 \%$ and $100 \%(p=0.87)$.

Long-term outcomes. The treatment outcomes in terms of the main endpoints of 3- and 5-year OS, LC and DFS for the RT group were as follows: $61 \%, 74 \%$ and $55 \%$, respectively and $53 \%, 69 \%$ and $47 \%$, respectively. In the CCRT group, 3-year OS, LC and DFS were $76 \%, 76 \%$ and $56 \%$, whilst for the neoCRT group they were $43 \%, 31 \%$ and $18 \%$. Owing to the shorter follow-up for the two latter groups, only the 3-year observations are reported.
The differences in the analyzed end-points were compared between the RT and CCRT groups because these groups included comparable patient populations. The differences were not statistically significant; however, with respect to OS, a statistical trend was observed (OS, $p=0.09$ ) (DFS, $p=0.93$ ) (LC, $p=0.59$ ). When the RT and CCRT groups were analyzed together, the 3- and 5-year OS rates were $66 \%$ and $58 \%$ while DFS rates were $56 \%$ and $47 \%$, respectively.

Figures 1 and 2 show OS and DFS for all groups.

\section{Discussion}

For muscle-invasive bladder cancer a trimodality treatment (TURB plus CRT) seems to be a good alternative to radical cystectomy $(2,4,6,11,12)$. Whether CCRT is better than surgery was not confirmed head-to-head and is probably not going to be confirmed in the future because of difficulties in accrual to such a kind of study (13). The pooled studies show, however, at least comparable results (14). 


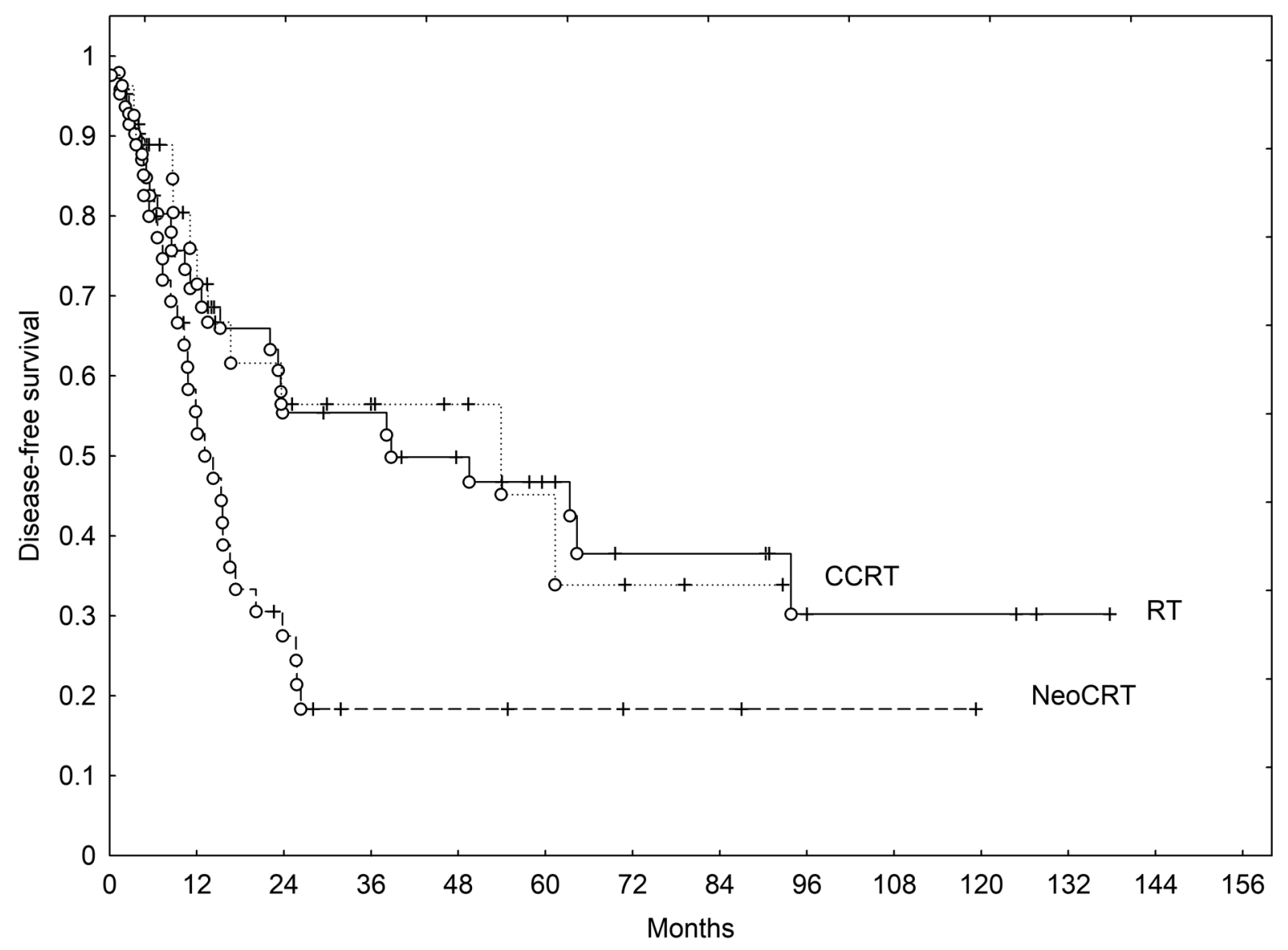

Figure 2. Disease-free survival of 27 patients treated with cisplatin-based chemoradiation (CCRT), 48 treated with radiation alone (RT), and 42 with locally advanced disease treated with neoadjuvant chemotherapy and radiation (neoCRT).

We found the results of a conservative approach with RT or CCRT gave 3- and 5-year OS rates of $66 \%$ and $58 \%$, which are comparable to those reported in other prospective and retrospective series $(6,11-12,15-18)$. For example, Lee et al. reported very similar OS and DFS of $50.8 \%$ and $39.9 \%$, with good toxicity profiles (15), as was also noted in our study. Murthy et al. reported 3-year OS and DFS rates of $67 \%$ and $66 \%$ (17), which our observations are also very similar to. Some studies reported an even better treatment outcome than that observed by us, with a 5 -year OS of $73 \%$ (19). Based on our experience and that of others, it may be stated that the results of a bladder-sparing approach may also be very good outside a clinical trial in general practice.

According to the experience from our center, we retained relatively high (64-66 Gy) total radiation doses when constructing the CCRT protocol. Although the clinical utility of pelvic lymph node irradiation was not confirmed in a randomized study, we used that approach because of the potential benefit (18). Irrespective of high total dose and pelvic irradiation, the tolerance of CCRT in the study group was very good. Radiation toxicity for this group was not increased in comparison to the RT group. Moreover, acute toxicity seemed even to be significantly less pronounced. The exact reason for this is not clear but it may be due to the more contemporary study period for CCRT and related technological capabilities such as image-guided RT, and dynamic techniques. The GU toxicity in the CCRT group was comparable to other modern image-guided intensitymodulated RT series, while GI toxicity in our study was either less pronounced or similar to others' experience (17, $18,20)$. It is important to point out that hematological toxicity in the CCRT group was excellent.

Interestingly, we found that RT alone after TURB gave a similar treatment outcome to CCRT. It should be emphasized, however, that the OS curves separated, favoring CCRT treatment with a statistical trend $(p=0.09)$. In the only two trials with randomization, there was no significant improvement of OS in the CRT arm, however, better locoregional control was observed $(2,5)$. On the other hand, the studies from Erlangen indicated an improvement of OS 
in the CCRT group over that treated with RT alone (6). However, this was not a randomized trial with some possible patient preselection. To overcome such preselection, we decided to include in the analysis those patients who had been treated with RT alone in the years before we implemented the tri-modality protocol into our practice. However, a drawback of such analysis was the difference in follow-up between the groups, and a possible influence of other confounding factors associated with progress in RT technology throughout the study period. The retrospective nature of the study and the small number of patients suggest that our results should be interpreted with caution.

Based on the relatively good results for RT alone, we also believe that there may be a subgroup of patients who can be treated with TURB and RT alone. Thus, our policy is to offer such patients CCRT, but in the case of their not being suitable for cisplatin chemotherapy, we may also offer RT alone.

Locally advanced bladder cancer is difficult to cure because of a substantial risk of occult dissemination and the probability that for large tumors, no pronounced regression occurs after RT or CRT. Therefore, neoadjuvant chemotherapy seems to be an attractive method. Local regression after neoadjuvant chemotherapy may help in the decision-making process, i.e. those patients who respond to neoadjuvant chemotherapy fare much better than nonresponders (21). It may also indicate which patients are good candidates for organ preservation (16). However, neoadjuvant chemotherapy is not used commonly by the RTOG group because RTOG 89-03 trial revealed substantial toxicity in the neoadjuvant arm, which resulted in the premature closure of the trial (22).

Some of the treatment results in the literature are better than those reported by us. However, it should be emphasized that those groups were composed of more favorable patients with muscle-invasive bladder cancer which should be rather compared with our CCRT group $(16,23,24)$. For instance, very good results were observed in a retrospective study by Perdona et al. (24) (5-year: $\mathrm{OS}=67.7 \%, \mathrm{LC}=56.3 \%)$.

Five-year OS of $30 \%$ was reported by Baxter et al. in a population with locally advanced bladder cancer treated with neoadjuvant CRT (25). Their observation is similar to ours. On the other hand, others reported the possibility of achieving better results, with a 5 -year DFS of $43 \%$ in patients with locally advanced bladder cancer (26). We speculate that neoadjuvant chemotherapy plus cystectomy might be a preferable option. However, if the main location of failure is distant, there may be no clear advantage of cystectomy over RT or CCRT, as also pointed out by others (16).

It can be expected that in approximately $15 \%$ of patients, disease will progress after neoadjuvant chemotherapy and they will not be candidates for further radical treatment - as was observed in our and others' studies $(16,26)$. This may be considered a drawback of neoadjuvant therapy. On the other hand, such patient suitability for upfront radical treatment and expectations towards long-term survival may be questionable. In contrast, the vast majority of patients with less advanced stages complete neoadjuvant chemotherapy and subsequent RT successfully $(23,24)$.

In another group of patients with advanced disease, with $22 \%$ of them presenting $\mathrm{N}+$ disease and $68 \%$ of patients with stage $\geq \mathrm{T} 3$, the 5 -year OS was $32 \%$ (27). The authors stated that a more effective systemic treatment might improve the treatment outcome. We could expect some improvement in treatment outcome with another, probably more efficacious, neoadjuvant chemotherapy schedule such as dose-dense MVAC $(1,28,29)$. Such a schedule was not used in our study. Nowadays more interest is placed on adjuvant therapy than on neoadjuvant, and some authors postulate better opportunities with such an approach (30). However, a direct comparison of two such treatment schedules has not yet been made in bladder cancer. Therefore, in a locally advanced setting for patients who are borderline candidates for radical treatment, it would be even more difficult to recommend one option over another.

\section{Conclusion}

The tolerance of CCRT was found to be very good, radiation side-effects were not exacerbated by the administration of concurrent cisplatin.

As long-term outcomes did not differ significantly between the RT and CCRT groups, and was satisfactory in both of them, in our opinion, RT alone after TURB may also be an option for properly selected patients who are not candidates for CCRT.

Due to a trend towards better OS in the group treated with CCRT, and taking into consideration the safety of such treatment in clinical practice, this is our recommended bladder-conserving approach.

The results of neoadjuvant chemotherapy and subsequent RT for locally advanced tumors are still not satisfactory, however, such treatment gives an opportunity of cure to some patients. More effort should be put into the selection of patients for radical treatment and improvement in its efficacy.

\section{Conflicts of Interest}

All Authors declare no conflicts of interest related to the study.

\section{Authors' Contributions}

All Authors contributed to the design of the study, acquisition and interpretation of the data. All Authors discussed, verified and approved the final version of the article. 


\section{References}

1 National Comprehensive Cancer Network. Bladder cancer (version 5.2018). Available at: https://www.ncen.org/ professionals/physician_gls/pdf/bladder.pdf [Last accessed on August $31^{\text {st }}$, 2020]

2 Coen JJ, Zhang P, Saylor PJ, Lee CT, Wu C-L, Parker W, Lautenschlaeger T, Zietman AL, Efstathiou JA, Jani AB, Kucuk O, Souhami L, Rodgers JP, Sandler HM and Shipley WU: Bladder preservation with twice-a-day radiation plus fluorouracil/cisplatin or once daily radiation plus gemcitabine for muscle-invasive bladder cancer: NRG/RTOG 0712-a randomized phase II trial. J Clin Oncol 37: 44-51, 2019. PMID: 3433852. DOI: $10.1200 / \mathrm{JCO} .18 .00537$

3 James ND, Hussain SA, Hall E, Jenkins P, Tremlett J, Rawlings C, Crundwell M, Sizer B, Sreenivasan T, Hendron C, Lewis R, Waters R, Huddart RA; BC2001 Investigators: Radiotherapy with or without chemotherapy in muscle invasive bladder cancer. N Eng J Med 366: 1477-1488, 2012. PMID: 22512481. DOI: 10.1056/NEJMoa1106106

4 Arcangeli G, Arcangeli S and Strigari L: A systematic review and meta-analysis of clinical trials of bladder-sparing trimodality treatment for muscle invasive bladder cancer (MIBC). Crit Rev Oncol Hematol 94: 105-115, 2015. PMID: 25541350. DOI: 10.1016/j.critrevonc.2014.11.007

5 Coppin CM, Gospodarowicz MK, James K, Tannock IF, Zee B, Carson J, Pater J and Sullivan LD: Improved local control of invasive bladder cancer by concurrent cisplatin and preoperative or definitive radiation. The National Cancer Institute of Canada Clinical Trials group. J Clin Oncol 14: 2901-2907, 1996. PMID: 8918486. DOI: 10.1200/JCO.1996.14.11.2901

6 Sauer R, Birkenhake S, Kühn R, Wittekind C, Schrott KM and Martus P: Efficacy of radiochemotherapy with platin derivatives compared to radiotherapy alone in organ-sparing treatment of bladder cancer. Int J Radiat Oncol Biol Phys 40: 121-127, 1998. PMID 9422567. DOI: 10.1016/s0360-3016(97)00579-8

7 McLaren DB: Neoadjuvant chemotherapy in transitional-cell carcinoma of the bladder. Clin Oncol 17: 503-507, 2005. PMID: 16238137. DOI: 10.1016/j.clon.2005.07.011

8 Cox JD, Stetz J and Pajak TF: Toxicity criteria of the Radiation Therapy Oncology Group (RTOG) and the European Organization for Research and Treatment of Cancer (EORTC). Int J Radiat Oncol Biol Phys 31: 1341-1346, 1995. PMID: 7713792. DOI: 10.1016/0360-3016(95)00060-C

9 Trotti A, Colevas AD, Setser A, Rush V, Jaques D, Budach V, Langer C, Murphy B, Cumberlin R, Coleman CN and Rubin P: CTCAE v3.0: development of a comprehensive grading system for the adverse effects of cancer treatment. Semin Radiat Oncol 13: 176-181, 2003. PMID: 12903007. DOI: 10.1016/S10534296(03)00031-6

10 Miszczyk M and Majewski W: Hematologic toxicity of conformal radiotherapy and intensity modulated radiotherapy in prostate and bladder cancer patients. Asian Pac J Cancer Prev 26: 2803-2806, 2018. PMID: 30360609. DOI: 10.22034/APJCP.2018.19.10.2803

11 Giacalone NJ, Shipley WU, Clayman RH, Niemierko A, Drumm M, Heney NM, Michaelson MD, Lee RJ, Saylor PJ, Wszolek MF, Feldman AS, Dahl DM, Zietman AL and Efstathiou JA: Long-term outcomes after bladder-preserving tri-modality therapy for patients with muscle-invasive bladder cancer: An updated analysis of the Massachusetts General Hospital experience. Eur Urol 71: 952-960, 2017. PMID: 28081860. DOI: 10.1016/j.eururo.2016.12.020

12 Mak RH, Hunt D, Shipley WU, Efstathiou JA, Tester WJ, Hagan MP, Kaufman DS, Heney NM and Zietman AL: Long-term outcomes in patients with muscle-invasive bladder cancer after selective bladder-preserving combined-modality therapy: A pooled analysis of Radiation Therapy Oncology group protocols 8802, 8903, 9506, 9706, 9906 and 0233. J Clin Oncol 32: 38013809, 2014. PMID: 25366678. DOI: 10.1200/JCO.2014.57.5548

13 Huddart RA, Birtle A, Maynard L, Beresford M, Blazeby J, Donovan J, Kelly JD, Kirkbank T, McLaren DB, Mead G, Moynihan C, Persad R, Scrase C, Lewis R and Hall E: Clinical and patient-reported outcomes of SPARE - a randomized feasibility study of selective bladder preservation versus radical cystectomy. BJU Int 120: 639-650, 2017. PMID: 28453896. DOI: $10.1111 /$ bju. 13900

14 Arcangeli G, Strigari L and Arcangeli S: Radical cystectomy versus organ-sparing trimodality treatment in muscle-invasive bladder cancer: A systematic review of clinical trials. Crit Rev Oncol Hematol 95: 387-396, 2015. PMID: 25934521. DOI: 10.1016/j.critrevonc.2015.04.006

15 Lee C-Y, Yang K-L, Ko H-L, Huang R-Y, Tsai P-P, Chen M-T, Lin Y-C, Hwang T I-S, Juang G-D and Chi K-H: Trimodality bladder-sparing approach without neoadjuvant chemotherapy for node-negative localized muscle-invasive urinary bladder cancer resulted in comparable cystectomy-free survival. Radiat Oncol 9: 213, 2014. PMID: 4261984. DOI: 10.1186/1748-717X-9-213

16 Hafeez S, Horwich A, Omar O, Mohammed K, Thompson A, Kumar P, Khoo V, Van As N, Eeles R, Dearneley D and Huddart $\mathrm{R}$ : Selective organ preservation with neo-adjuvant chemotherapy for the treatment of muscle-invasive transitional cell carcinoma of the bladder. Br J Cancer 112: 1626-1635, 2015. PMID: 25897675. DOI: $10.1038 /$ bjc. 2015.109

17 Murthy V, Masodkar R, Kalyani N, Mahantshetty U, Bakshi G, Prakash G, Joshi A, Prabhash K, Ghonge S and Shrivastava S: Clinical outcomes with dose-escalated adaptive radiation therapy for urinary bladder cancer: A prospective study. Int J Radiat Oncol Biol Phys 94: 60-66, 2016. PMID: 26547385. DOI: 10.1016/j.ijrobp.2015.09.010

18 Tunio MA, Hashmi A, Qayyum A, Mohsin R and Zaeem A: Whole-pelvis or bladder only chemoradiation for lymph nodenegative invasive bladder cancer: Single-institution experience. Int J Radiat Oncol Biol Phys 82: e457-462, 2012. PMID: 21945107. DOI: 10.1016/j.ijrobp.2011.05.051

19 Zapatero A, De Vidales CM, Arellano R, Ibanez Y, Bocardo G, Perez M, Rabadan M, Vicente FG, Conde JAC and Olivier C: Long-term results of two prospective bladder-sparing trimodality approaches for invasive bladder cancer: Neoadjuvant chemotherapy and concurrent radio-chemotherapy. Urology 80: 1056-1062, 2012. PMID: 22999456. DOI: 10.1016/j.urology.2012.07.045

20 Efstathiou J, Bae K, Shipley WU, Kaufman DS, Hagan MP, Heney NM and Sandler HM: Late pelvic toxicity after bladdersparing therapy in patients with invasive bladder cancer: RTOG 89-03, 95-06, 97-06, 99-06. J Clin Oncol 27: 4055-4061, 2009. PMID: 19636019. DOI: 10.1200/JCO.2008.19.5776

21 Petrelli F, Coinu A, Cabiddu M, Ghilardi M, Vavassiri I and Barni S: Correlation of pathologic complete response with survival after neoadjuvant chemotherapy in bladder cancer treated with cystectomy: a meta-analysis. Eur Urol 65: 350-357, 2014. PMID: 23849998. DOI: 10.1016/j.eururo.2013.06.049 
22 Shipley WU, Winter KA, Kaufman DS, Lee WR, Heney NM, Tester WR, Donnelly BJ, Venner PM, Perez CA, Murray KJ, Doggett RS and True LD: Phase III trial of neoadjuvant chemotherapy in patients with invasive bladder cancer treated with selective bladder preservation by combined radiation therapy and chemotherapy: initial results of Radiation Therapy Oncology group 89-03. J Clin Oncol 16: 3576-3583, 1998. PMID: 9817278. DOI: 10.1200/JCO.1998.16.11.3576

23 Jiang DM, Jiang H, Chung PWM, Zlotta AR, Fleshner NE, Bristow RG, Berlin A, Kulkarni GS, Alimohamed NS, Lo G and Sridhar SS: Neoadjuvant chemotherapy before bladder-sparing chemoradiotherapy in patients with nonmetastatic muscleinvasive bladder cancer. Clin Genitourin Cancer 17: 38-45, 2019. PMID: 30686350. DOI: 10.1016/j.clgc.2018.09.021

24 Perdona S, Autorino R, Damiano R, De Sio M, Morrica B, Gallo L, Silvestro G, Farella A, De Placido S and Di Lorenzo G: Bladder-sparing combined-modality approach for muscleinvasive bladder cancer. A multi-institutional long-term experience. Cancer 112: 75-83, 2008. PMID: 18008364. DOI: 10.1002/cncr.23137

25 Baxter E, Dennis K, Kollmannsberger C, Black P, Attwell A, Morris WJ and Tyldesley S: Radical trimodality therapy for patients with locally advanced bladder cancer: The British Columbia Cancer Agency experience. Urol Oncol 33: 66 e1319, 2015. PMID: 25168459. DOI: 10.1016/j.urolonc.2014.07.009

26 Serretta V, Lo Greco G, Pavone C and Pavone-Macaluso M: The fate of patients with locally advanced treated bladder cancer treated conservatively with neoadjuvant chemotherapy, extensive transurethral resection and radiotherapy: 10-year experience. J Urol 159: 1187-1191, 1998. PMID: 9507829.
27 Hussain MH, Glass TR, Forman J, Sakr W, Smith DC, Al-Sarraf M, Jones J, Balcerzak SP, Crawford ED and Grossman HB: Combination cisplatin 5-fluororacil and radiation therapy for locally advanced unresectable or medically unfit bladder cancer cases: A Southwest Oncology Group study. J Urol 165: 56-60, 2001. PMID: 11125363. DOI: 10.1097/00005392-200101000-00014

28 Sternberg CN, de Mulder P, Schornagel JH, Theodore C, Fossa $\mathrm{SD}$, van Oosterom AT, Witjes JA, Spina M, van Groeningen CJ, Duclos B, Roberts JT, de Balincourt C, Collette L and EORTC Genito-Urinary Cancer Group: Seven year update of an EORTC phase III trial of high-dose intensity M-VAC chemotherapy and G-CSF versus classic M-VAC in advanced urothelial tract tumours. Eur J Cancer 42: 50-54, 2006. PMID: 16330205. DOI: 10.1016/j.ejca.2005.08.032

29 Zargar H, Shah JB, van Rhijn BW, Daneshmand S, Bivalacqua TJ, Spiess PE, Black PC, Kassouf $\mathrm{W}$ and Collaborators: Neoadjuvant dose dense MVAC versus gemcitabine and cisplatin in patients with cT3-4aNOM0 bladder cancer treated with radical cystectomy. J Urol 199: 1452-1458, 2018. PMID: 29329894. DOI: $10.1016 /$ j.juro.2017.12.062

30 Joseph $\mathrm{N}$ and Choudhury A: Adjuvant chemotherapy is more suitable than neoadjuvant chemotherapy for muscle-invasive bladder cancer patients treated with radical chemoradiotherapy. Int J Radiat Oncol Biol Phys 96: 614-616, 2016. PMID: 27681756. DOI: $10.1016 /$ j.ijrobp.2016.06.2452

Received May 11, 2020

Revised August 31, 2020

Accepted September 1, 2020 